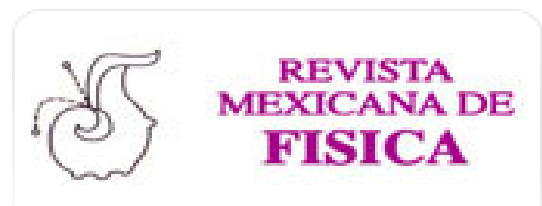

Revista Mexicana de Física

ISSN: 0035-001X

rmf@ciencias.unam.mx

Sociedad Mexicana de Física A.C.

México

López, G.; González, G.

Quantum bouncer with dissipation

Revista Mexicana de Física, vol. 52, núm. 2, diciembre, 2006, pp. 126-131

Sociedad Mexicana de Física A.C.

Distrito Federal, México

Available in: http://www.redalyc.org/articulo.oa?id=57065604

How to cite

Complete issue

- More information about this article

Journal's homepage in redalyc.org

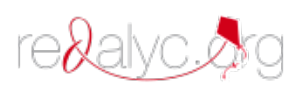

Scientific Information System Network of Scientific Journals from Latin America, the Caribbean, Spain and Portugal Non-profit academic project, developed under the open access initiative 


\title{
Quantum bouncer with dissipation
}

\author{
G. López and G. González \\ Departamento de Física de la Universidad de Guadalajara, \\ Apartado Postal 4-137, 44410 Guadalajara, Jalisco, México.
}

Recibido el 25 de abril de 2005; aceptado el 17 de enero de 2006

\begin{abstract}
Effects on the spectra of the quantum bouncer due to dissipation are given when a linear o quadratic dissipation in the velocity of the particle is taken into account. Classical constants of motion and Hamiltonians are deduced for these systems and their quantized eigenvalues are estimated through perturbation theory. Differences were found comparing the eigenvalues of the constants of motion and the eigenvalues of the Hamiltonians. The cases when the dissipation parameters go to zero are compared with the nondissipative cases.
\end{abstract}

Keywords: Classical mechanics; discrete systems; formalism.

Los efectos en el espectro del rebotador cuántico debido a la disipación son dados cuando una disipación lineal o cuadrática en la velocidad de la partícula es tomada en cuenta. Constantes de movimiento clásicas y Hamiltonianos se deducen para estos sistemas y sus eigenvalores cuantizados son estimados mediante la teoría de perturbaciones. Se encuentran diferencias comparando los eigenvalores de las constantes de movimiento y los eigenvalores de los Hamiltonianos. Los casos cuando los parámetros de disipación se hacen cero son comparados con los casos no disipativos.

Descriptores: Mecánica clásica; sistemas discretos; formalismo.

PACS: 03.20.+i; 03.65.Ca

\section{Introduction}

Dissipative systems have been one of the must subtle and difficult topics to deal with in classical [1] and quantum physics [2]. In general, constructing a consistent Lagrangian and Hamiltonian formulation for a given dissipative system can be a big challenge [3]. There are basically two approaches to studying dissipative systems. The first one tries to bring about dissipation as a result of averaging over all the coordinates of the bath system, where one considers the whole system as composed of two parts, our original conservative system and the bath system, which interacts with the conservative system and causes the dissipation (of energy) in it [4]. This approach has its own value and will not be followed or discussed here. The second approach considers that the bath system produces an average effect on our initially conservative system which is expressed as an additional external velocity-dependent force acting on the conservative system and transforming it into a dissipative system with this velocity-dependent force. The resulting classical dissipative system thus contains this phenomenological (or theoretical) velocity-dependent force. Then, the question arises over its consistent Lagrangian and Hamiltonian formalism and the consequences of its quantization. This approach, in addition, allows us to study and test the Hamiltonian approach for quantum mechanics and its consistency [5], and is the approach we will follow in this paper. A system which has attracted our attention for dissipation study through the above approach is the quantum bouncer. The quantum bouncer [6] is the quantization of the motion of a particle which is attracted by the constant gravity force, that is, close to surface of the earth. This particle hits a perfectly reflecting surface, producing the bouncing effect. This system, with an additional dissipation force, is particular importance because of its potential experimental realization. This dissipative system has been studied very little, until now using the first approach mentioned above [7].

We will assume that the external velocity-dependent force has linear and quadratic dependence with respect to the velocity. This approach gives us the opportunity to check the nature of quantization using the Hamiltonian or constant of motion associated with the system, that is, using the usual quantization of the generalized linear momentum or using the quantization of the velocity. This consideration is particularly interesting in dissipative systems, since one can not always find a Hamiltonian as a function of the variable position and linear momentum [8]; that is, velocity "v" can not always be known explicitly in terms of linear momentum "p" and position " $\mathrm{x}$ " of the particle through the relation $p=\partial L / \partial v$, where $L$ is the Lagrangian of the system. This paper is organized as follows: we present the classical study for the dissipative system considering the linear and quadratic velocitydependent force. The constant of motion, the Lagrangian, and the Hamiltonian of the system are derived, and we give their expressions up to second order in the dissipation parameter. We present the modification for the eigenvalues of the quantum bouncer, when this dissipation is taken into account, for the above approximated (weak dissipation) constant of motion and Hamiltonian using quantum perturbation theory. Finally, conclusions and some discussions of our results are given.

\section{Classical linear dissipation}

The motion of a particle of mass $m$ under a constant gravitational force and a linear dissipative force is described by the equation

$$
m \frac{d^{2} x}{d t^{2}}=-m g-\alpha v
$$


where $x$ is the position of the particle, $g$ is the constant acceleration due to the earth's gravity, $\alpha$ is the parameter which characterizes the dissipation, and $v=d x / d t$ is the velocity of the particle. A constant of motion of the autonomous system (1) is a function $K_{\alpha}=K_{\alpha}(x, v)$ satisfying the equation [9]

$$
v \frac{\partial K_{\alpha}}{\partial x}-\left(g+\frac{\alpha}{m} v\right) \frac{\partial K_{\alpha}}{v}=0 .
$$

The solution of this equation, such that

$$
\lim _{\alpha \rightarrow 0} K_{\alpha}=m v^{2} / 2+m g x
$$

(the usual total energy for the non dissipative system), is given by

$$
K_{\alpha}=\frac{m^{2} g v}{\alpha}-m\left(\frac{m g}{\alpha}\right)^{2} \ln \left(1+\frac{\alpha v}{m g}\right)+m g x .
$$

The Lagrangian associated with (1) can be obtained using the known expression [5]

$$
L_{\alpha}=v \int \frac{K_{\alpha}(x, v)}{v^{2}} d v
$$

producing the following Lagrangian:

$$
\begin{aligned}
L_{\alpha}= & \frac{m^{2} g v}{\alpha} \ln \left(1+\frac{\alpha v}{m g}\right) \\
& +m\left(\frac{m g}{\alpha}\right)^{2} \ln \left(1+\frac{\alpha v}{m g}\right)-m g x-\frac{m^{2} g v}{\alpha} .
\end{aligned}
$$

Therefore, the generalized linear momentum and Hamiltonian are given by

$$
p_{\alpha}=\frac{m^{2} g}{\alpha} \ln \left(1+\frac{\alpha v}{m g}\right)
$$

and

$$
H_{\alpha}=m\left(\frac{m g}{\alpha}\right)^{2}\left(\exp \left(\frac{\alpha p_{\alpha}}{m^{2} g}\right)-1\right)-\frac{m g}{\alpha} p_{\alpha}+m g x .
$$

At two orders in the dissipation parameter $\alpha$, one has the constant of motion, the Lagrangian, the generalized linear momentum, and Hamiltonian given as

$$
\begin{aligned}
K & =\frac{1}{2} m v^{2}+m g x-\frac{\alpha}{3 g} v^{3}+\frac{\alpha^{2}}{4 m g^{2}} v^{4}, \\
L & =\frac{1}{2} m v^{2}-m g x-\frac{\alpha}{6 g} v^{3}+\frac{\alpha^{2}}{12 m g^{2}} v^{4}, \\
p & =m v-\frac{\alpha}{2 g} v^{2}+\frac{\alpha^{2}}{3 m g} v^{3},
\end{aligned}
$$

and

$$
H=\frac{p^{2}}{2 m}+m g x+\frac{\alpha}{6 m g} p^{3}+\frac{\alpha^{2}}{24 m^{5} g^{2}} p^{4} .
$$

One should note that all these quantites go to the nondissipative case when the dissipative parameter goes to zero. The constant of motion (3) or (8a) and the Hamiltonian (7) or $(8 \mathrm{~d})$ bring about the damping bouncing effect on spaces $(x, v)$ and $(x, p)$. The dissipative parameter $\alpha$ can be determined by measuring the velocity $v_{o}$ at the reflecting surface $(x=0)$ and then measuring its maximum displacement $x_{\max }$ $(v=0)$. By equaling the value of the constant of motion in both situations, one obtains the expression

$$
\frac{m^{2} g v_{o}}{\alpha}-m\left(\frac{m g}{\alpha}\right)^{2} \ln \left(1+\frac{\alpha v_{o}}{m g}\right)=m g x_{\max },
$$

where the parameter $\alpha$ can be found.

\section{Classical quadratic dissipation}

In this case, the motion of the particle is described by the equation

$$
m \frac{d^{2} x}{d t^{2}}=-m g-\gamma v|v|,
$$

where $\gamma$ represents a dissipation constant which, of course, is different from the previous case. Proceeding in the same way as we did for the linear case, the constant of motion, Lagrangian, generalized linear momentum, and Hamiltonian are given by

$$
\begin{aligned}
K_{ \pm} & =\frac{1}{2} m v^{2} \exp \left( \pm \frac{2 \gamma x}{m}\right) \pm \frac{m^{2} g}{2 \gamma}\left(\exp \left( \pm \frac{2 \gamma x}{m}\right)-1\right) \\
L_{ \pm} & =\frac{1}{2} m v^{2} \exp \left( \pm \frac{2 \gamma x}{m}\right) \mp \frac{m^{2} g}{2 \gamma}\left(\exp \left( \pm \frac{2 \gamma x}{m}\right)-1\right) \\
p_{ \pm} & =m v \exp \left( \pm \frac{2 \gamma x}{m}\right)
\end{aligned}
$$

and

$$
H_{ \pm}=\frac{p_{ \pm}^{2}}{2 m} \exp \left(\mp \frac{2 \gamma x}{m}\right) \pm \frac{m^{2} g}{2 \gamma}\left(\exp \left( \pm \frac{2 \gamma x}{m}\right)-1\right),
$$

where the upper sign corresponds to the case $v \geq 0$, and the lower sign corresponds to the case $v<0$. These equations were already given in Ref. 10, and the nondissipative case is obtained when the dissipative parameter goes to zero. The damping effect of the bouncing particle in the space $(x, v)$ can be traced in the following way: starting with the initial condition $x_{o}=0$ and $v_{o}>0$, for example, the constant of motion $K_{+}$is determined, $K_{+}=m v_{o}^{2} / 2$. Then, the maximum distance $x_{\max }(v=0)$ is calculated from the expression $K_{+}=\left(m^{2} g / 2 \gamma\right)\left(\exp \left(2 \gamma x_{\max } / m\right)-1\right)$, which helps to calculate the constant $K_{-}$,

$$
K_{-}=-\left(m^{2} g / 2 \gamma\right)\left(\exp \left(-2 \gamma x_{\max } / m\right)-1\right) .
$$

This $K_{-}$is now used to calculate the velocity at the turning point $(x=0), v_{1}^{*}=-\sqrt{2 K_{-} / m}$. Considering a perfectly reflexing surface, the velocity of the bouncing particle 
for the next cycle is $v_{1}=-v_{1}^{*}\left(v_{1}<v_{o}\right)$, and the above cycle is reproduced again, and so on. Starting with the same initial conditions, the trajectories in this space are one below the other at any time, as the damping factor is greater. The damping effect in the space $(x, p)$ can be analyzed similarly through the Hamiltonian approach. However, the trajectories starting with the same initial conditions in this space are not below the other all the time, as the damping factor is greater. This strange effect is due to the change in sign in (11d) with respect to (11a), produced by the position and velocity dependence of the expression (11c).

To determine the constant $\gamma$ through the constant of motion, one can start with the initial conditions $\left(x_{o}=0, v_{o}>0\right)$ and can determine the constant of motion $K_{+}=m v_{o}^{2} / 2$. Then, one can measure the maximum displacement $x_{\max }$ $(v=0)$ and solve $\gamma$ from the equation

$$
\frac{1}{2} m v_{o}^{2}=\frac{m^{2} g}{2 \gamma}\left(\exp \left(\frac{2 \gamma x_{\max }}{m}\right)-1\right) .
$$

Up to second order in the dissipation parameter, one has, from (11a) to (11d) the constant of motion, the Lagrangian, the generalized linear momentum, and the Hamiltonian given by

$$
\begin{gathered}
K_{ \pm}=\frac{1}{2} m v^{2}+m g x \pm \gamma\left[v^{2} x+g x^{2}\right] \\
+\gamma^{2}\left[v^{2} x^{2} / m+2 g x^{3} / 3 m\right], \\
L_{ \pm}=\frac{1}{2} m v^{2}-m g x \pm \gamma\left[v^{2} x-g x^{2}\right] \\
+\gamma^{2}\left[v^{2} x^{2} / m-2 g x^{3} / 3 m\right], \\
H_{ \pm}=\frac{p^{2}}{2 m}+m g x \mp \gamma[2 v x]+\gamma^{2}\left[2 v x^{2} / m\right], \\
+\gamma^{2}\left[p^{2} x^{2} / m^{3}+2 g x^{3} / 3 m\right] .
\end{gathered}
$$

\section{Quantization of the constant of motion}

Equations (8a) and (13a) can be written as

$$
K(x, v)=K_{o}(x, v)+V(x, v),
$$

where $K_{o}$ is the constant of motion without dissipation

$$
K_{o}(x, v)=\frac{1}{2} m v^{2}+m g x,
$$

and $V$ takes into account the dissipation factors

$$
V(x, v)= \begin{cases}-\alpha\left(\frac{v^{3}}{3 g}\right)+\alpha^{2}\left(\frac{v^{4}}{4 m g^{2}}\right) & \text { (linear case) } \\ \mp \gamma\left[v^{2} x+g x^{2}\right]+\gamma^{2}\left[\frac{v^{2} x^{2}}{m}+\frac{2 g x^{3}}{3 m}\right] & \text { (quadratic case) }\end{cases}
$$

The quantization of (14) can be carried out through the associated Schrödinger's equation of this constant of motion

$$
i \hbar \frac{\partial \Psi}{\partial t}=\widehat{K}(\widehat{x}, \widehat{v}) \Psi
$$

where $\Psi=\Psi(x, t)$ is the wave function, $\hbar$ is the Plank constant divided by $2 \pi, \widehat{K}=\widehat{K}_{o}+\widehat{V}$ is a Hermitian operator associated to (17), and $\widehat{v}$ is the velocity operator defined as

$$
\widehat{v}=-\frac{i \hbar}{m} \frac{\partial}{\partial x} \text {. }
$$

Since Eq. (16) represents a stationary problem, the usual proposition $\Psi(x, t)=\exp \left(-i E^{K} t / \hbar\right) \psi(x)$ transforms (16) to an eigenvalue problem:

$$
\left(\widehat{K}_{o}+\widehat{V}\right) \psi=E^{K} \psi
$$

Taking the operator $\widehat{V}$ as a perturbation of the constant of motion $K_{o}$, one can calculate an approximate solution to the problem (18) through perturbation theory. The solution of the eigenvalue problem

$$
\widehat{K}_{o} \psi_{n}^{(0)}=E_{n}^{(0)} \psi_{n}^{(0)}
$$

is well known [6], with $\psi_{n}^{(0)}$ being the eigenfunction given by

$$
\psi_{n}^{(0)}=\frac{A i\left(z-z_{n}\right)}{\left|A i^{\prime}\left(-z_{n}\right)\right|}
$$

where $A i$ and $A i^{\prime}$ are the Airy function and its first differentiation, and $z_{n}$ is its nth-zero $\left(\operatorname{Ai}\left(-z_{n}\right)=0\right)$ which ocurres for a negative argument only. $z$ is the normalized variable $z=x / l_{g}$ with $l_{g}=\left(\hbar^{2} / 2 m^{2} g\right)^{1 / 3}$, and $z_{n}$ is related to the eigenvalue $E_{n}^{(0}$ through the expression

$$
z_{n}=\frac{E_{n}^{(0)}}{m g l_{g}} .
$$

Up to second order in perturbation theory, the eigenvalues of (18) are given (in Dirac notation [11]) as

$$
E_{n}^{K}=E_{n}^{(0)}+\langle n|\widehat{V}| n\rangle+\sum_{k \neq n} \frac{|\langle n|\widehat{V}| k\rangle|^{2}}{E_{k}^{(0)}-E_{n}^{(0)}},
$$


where $\langle z \mid n\rangle=\psi_{n}^{(0)}$. Using the Hermitian operators

$$
\widehat{v^{2} x}=\left(\widehat{v}^{2} x+\widehat{v} x \widehat{v}+x \widehat{v}^{2}\right) / 3
$$

$$
\left\langle n\left|x^{s}\right| k\right\rangle=l_{g}^{s}\left\langle n\left|z^{s}\right| k\right\rangle
$$

and

$$
\widehat{v^{2} x^{2}}=\left(\widehat{v}^{2} x^{2}+\widehat{v} x^{2} \widehat{v}+x^{2} \widehat{v}^{2}+x \widehat{v}^{2} x+x \widehat{v} x \widehat{v}+\widehat{v} x \widehat{v} x\right) / 6
$$

and

for the associated expressions on (25b), and using the relations

$$
\left\langle n\left|d^{s} / d x^{s}\right| k\right\rangle=l_{g}^{-s}\left\langle n\left|d^{s} / d z^{s}\right| k\right\rangle
$$

for any integer $s$, one has (see appendix for a list of matrix elements)

$$
E_{n}^{K}=E_{n}^{(0)}+ \begin{cases}\alpha^{2}\left[\frac{l_{g}^{2} z_{n}^{2}}{5 m}+\frac{8}{9} g l_{g}^{3} \sum_{k \neq n} \frac{\left|1 / 2+m g l_{g} /\left(E_{k}^{(0)}-E_{n}^{(0)}\right)\right|^{2}}{E_{k}^{(0)}-E_{n}^{(0)}}\right], \quad \text { (linear) } \\ \mp \gamma \frac{12 g l_{g}^{2} z_{n}^{2}}{15}+\gamma^{2}\left[\left(-\frac{1}{2}+\frac{56 z_{n}^{3}}{105}\right) \frac{2 g l_{g}^{3}}{m}+4 g^{2} l_{g}^{4} \sum_{k \neq n} a_{n k}\right], & \text { (quadratic) }\end{cases}
$$

where $a_{n k}$ is a real number given by

$$
a_{n k}=\frac{\left|12-2 z_{k}\left(z_{n}-z_{k}\right)^{2}+\left(z_{n}-z_{k}\right)^{3}\right|^{2}}{\left(z_{k}-z_{n}\right)^{9}} .
$$

Note that for the linear dissipative case, there is no real contribution at a first approximation, and for the quadratic dissipative case, the first order contribution depends on whether the particle is moving up (-) or down (-). Within a full cycle, this first order correction is cancelled out and the second order contribution remains. Of course, for the approximation (23a) to be valid, one must assume that the second term of this expression must be much less than $E_{n}^{(0)}$ which makes a restriction on the possible value of the dissipative parameter. Of course, when the parameters of dissipation go to zero, one gets the nondissipative eigenvalues.

\section{Quantization of the Hamiltonian}

Equations (8d) and (13d) can be written as

$$
H(x, p)=H_{o}(x, p)+W(x, p),
$$

where $H_{o}$ is the Hamiltonian without dissipation,

$$
H_{o}(x, p)=\frac{p^{2}}{2 m}+m g x,
$$

and $W$ has the dissipation terms,

$$
W(x, p)=\left\{\begin{array}{l}
\alpha\left(\frac{p^{3}}{6 m g}\right)+\alpha^{2}\left(\frac{p^{4}}{24 m^{5} g^{2}}\right) \quad \text { (linear) } \\
\mp \gamma\left[\frac{p^{2} x}{m^{2}}-g x^{2}\right]+\gamma^{2}\left[\frac{p^{2} x^{2}}{m^{3}}+\frac{2 g x^{3}}{3 m}\right] \quad \text { (quadratic) }
\end{array}\right.
$$

It is necessary to mention that the quantization of some systems for quadratic dissipation has been solved by different authors $[10,12]$ but perfectly reflexing wall potential,

$$
\tilde{V}(x)=\left\{\begin{array}{ll}
\infty & \text { for } x<0 \\
m g x & \text { for } x \geq 0
\end{array} .\right.
$$

Moreover, the solution given in Ref. 10 is singular when the dissipation parameter goes to zero. Therefore, we think it worth while to make analysis of the quantization for small orders in the parameter $\gamma$. For the usual Shrödinger quantization approach, one has the stationary equation

$$
i \hbar \frac{\partial \Psi}{\partial t}=\widehat{H}(x, \widehat{p}) \Psi
$$

where $\widehat{H}$ is the Hamiltonian operator associated to (24), and $\widehat{p}$ is the usual linear momentum operator $\widehat{p}=-i \hbar \partial / \partial x$. Equation (27) is transformed to an eigenvalue problem, $\widehat{H} \psi(x)=E^{H} \psi(x)$, through the proposition $\Psi(x, t)=\exp \left(-i E^{H} t / \hbar\right) \psi(x)$, since the Hamiltonian $\widehat{H}$ is given by $\widehat{H}=\widehat{H}_{o}+\widehat{W}$, where the solution of the equation

$$
\widehat{H}_{o} \psi_{n}^{(0)}=E_{n}^{(0)} \psi_{n}^{(0)}
$$


is given by (20) and (21). Perturbation theory can be used to determine the approximate values of the eigenvalues $E_{n}^{H}$ (expression (22)). Using the Hermitian operators

$$
\widehat{p^{2} x}=\left(\widehat{p}^{2} x+\widehat{p} x \widehat{p}+x \widehat{p}^{2}\right) / 3
$$

and

$$
\widehat{p^{2} x^{2}}=\left(\widehat{p}^{2} x^{2}+\widehat{p} x^{2} \widehat{v}+x^{2} \widehat{p}^{2}+x \widehat{p}^{2} x+x \widehat{p} x \widehat{p}+\widehat{p} x \widehat{p} x\right) / 6
$$

for the associated expressions on (25b), one gets

$$
E_{n}^{H}=E_{n}^{(0)}+\left\{\begin{array}{l}
\alpha^{2}\left[\frac{l_{g}^{2} z_{n}^{2}}{30 m}+\frac{4}{9} g l_{g}^{3} \sum_{k \neq n} \frac{\left|1 / 2+m g l_{g} /\left(E_{k}^{(0)}-E_{n}^{(0)}\right)\right|^{2}}{E_{k}^{(0)}-E_{n}^{(0)}}\right] \quad \text { (linear) } \\
\pm \gamma \frac{4 g l_{g}^{2} z_{n}^{2}}{15}+\gamma^{2}\left[\left(-\frac{1}{2}+\frac{56 z_{n}^{3}}{105}\right) \frac{2 g l_{g}^{3}}{m}+4 g^{2} l_{g}^{4} \sum_{k \neq n} a_{n k}\right], \quad \text { (quadratic) }
\end{array}\right.
$$

where $a_{n k}$ is given by (23b). One must note here too that, when the parameters of dissipation go to zero, one gets the usual eigenvalues for the nondissipative system. As one can see from (23) and (29), there is a difference between the eigenvalues associated with the constant of motion and those associated with the Hamiltonian. Their relative difference, $\delta E_{n}=\left(E_{n}^{H}-\right.$ $\left.E_{n}^{K}\right) / E_{n}^{(0)}$, is given by

$$
\frac{\delta E_{n}}{E_{n}^{(0)}}=\left\{\begin{array}{l}
\alpha^{2}\left[-\frac{1}{6} \frac{l_{g} z_{n}}{6 m^{2} g}-\frac{4}{9} \frac{l_{g}^{2}}{m z_{n}} \sum_{k \neq n} \frac{\left|1 / 2+m g l_{g} /\left(E_{k}^{(0)}-E_{n}^{(0)}\right)\right|^{2}}{E_{k}^{(0)}-E_{n}^{(0)}}\right] \quad \text { (linear) } \\
\pm \gamma \frac{16 g l_{g}^{2} z_{n}^{2}}{15},
\end{array}\right.
$$

\section{Conclusion}

The classical and quantum problem of a particle bouncing on a hard surface under the influence of gravity and subject to a linear and quadratic velocity dissipative force were treated using the constant of motion and the Hamiltonian of the system. Expressions (3) and (11a) give us the expected damping behavior of the particle in the $(x, v)$ space, but the expressions (7) and (11d) show an unexpected behavior in the $(x, p)$ space (two trajectories in this space, for dissipative parameters one bigger than the other, do not follow one under the other all the time). In addition, all our expressions are reduced to the nondissipative case when the parameters of dissipation go to zero. For the quantum case, we have analyzed the eigenvalues for the constant of motion and Hamiltonian up to the second order in the dissipation parameter using perturbation theory. Relation (30) tells us that that there is a difference whether the constant of motion or the Hamiltonian is quantized, and it suggests that maybe one could see this difference experimentally. Finally, one must observe that for the full linear case (7), it is possible to solve exactly the Shrödinger equation in the momentum representation, and this will be analyzed in a future paper.

\section{Appendix}

We show here a list of some matrix elements from Ref. 6 and some others calculated from the same reference (a correction of sign has been made to some matrix elements). Given the functions (20) and $n \neq k$, one has

$$
\begin{aligned}
\langle n \mid k\rangle & =\delta_{n k} & & \\
\langle n|z| n\rangle & =\frac{2}{3} z_{n} & \langle n|z| k\rangle & =\frac{2(-1)^{n+k+1}}{\left(z_{n}-z_{k}\right)^{2}} \\
\left\langle n\left|z^{2}\right| n\right\rangle & =\frac{8}{15} z_{n}^{2} & \left\langle n\left|z^{2}\right| k\right\rangle & =\frac{24(-1)^{n+k+1}}{\left(z_{n}-z_{k}\right)^{4}}
\end{aligned}
$$




$$
\begin{aligned}
\left\langle n\left|z^{3}\right| n\right\rangle & =\frac{3}{7}+\frac{48}{105} z_{n}^{3} & \left\langle n\left|z^{3}\right| k\right\rangle & =\frac{24\left(z_{n}+z_{k}\right)(-1)^{n+k+1}}{\left(z_{n}-z_{k}\right)^{4}} \\
\left\langle n\left|\frac{d}{d z}\right| n\right\rangle & =0 & \left\langle n\left|\frac{d}{d z}\right| k\right\rangle & =\frac{(-1)^{n+k}}{z_{n}-z_{k}} \\
\left\langle n\left|\frac{d^{2}}{d z^{2}}\right| n\right\rangle & =-\frac{1}{3} z_{n} & \left\langle n\left|\frac{d^{2}}{d z^{2}}\right| k\right\rangle & =\frac{2(-1)^{n+k}}{\left(z_{n}-z_{k}\right)^{2}} \\
\left\langle n\left|\frac{d^{3}}{d z^{3}}\right| n\right\rangle & =\frac{1}{2} & \left\langle n\left|\frac{d^{3}}{d z^{3}}\right| k\right\rangle & =\left(\frac{1}{2}+\frac{1}{z_{k}-z_{n}}\right)(-1)^{n+k} \\
\left\langle n\left|\frac{d^{4}}{d z^{4}}\right| n\right\rangle & =\frac{1}{5} z_{n}^{2} & \left\langle n\left|\frac{d^{4}}{d z^{4}}\right| k\right\rangle & =\frac{-2\left(z_{k}-z_{n}\right)+24-2 z_{k}\left(z_{k}-z_{n}\right)^{2}}{\left(z_{k}-z_{n}\right)^{4}}(-1)^{n+k}
\end{aligned}
$$

1. R. Glauber and V.I. Man'ko, Sov. Phys. JEPT 60 (1984) 450; V.V. Dodonov, Hadron J. 4 (1981) 173; G. López, M. Murgía, and M. Sosa, Mod. Phys. Lett. B 11 (1997) 625.

2. M. Razavy, Can. J. Phys. 50 (1972) 2037; S. Okubo, Phys. Rev. A 23 (1981) 2776; G. López, M. Murgía, and M. Sosa, Mod. Phys. Lett. B 15 (2001) 965.

3. M. Mijatovic, B. Veljanoski, and D. Hajdukovic, Hadronic J. 7 (1984) 1207; G. López, Ann. Phys. 251 (1996) 372; G. López and G. González, IL Nuo. Cim. B, 118 (2003) 107.

4. A.O. Caldeira and A.T. Leggett, Physica A 121 (1983) 587; W.G. Unruh and W.H. Zurek, Phys. Rev. D 40 (1989) 1071; B.L. Hu, J.P. Paz, and Y. Zhang, Phys. Rev. D 45 (1992) 2843; G.P. Berman, F. Borgonovi, G.V. López, and V.I. Tsifrinovich, Phys. Rev. A 68 (2003) 012102.

5. G. López, Rev. Mex. Fís. 48 (2002) 10.

6. J. Gean-Banacloche, Am. J. Phys. 67 (1999) 776; D.M. Goodmanson, Am. J. Phys. 68 (2000) 866.
7. R. Onofrio and L. Viola, quant-ph/9606024 v1 (1996).

8. G. López, Rev. Mex. Fís. 45 (1999) 1817.

9. G. López, Partial Differential Equations of First Order and Their Applications to Physics (World Scientific, 1999) p. 37.

10. F. Negro and A. Tartaglia, Phys. Lett. A 77 (1980); F. Negro and A. Tartaglia, Phys. Rev. A 23 (1981) 1591.

11. P.A.M. Dirac, The Principles of Quantum Mechanics (Oxford Science Publications, 1992).

12. J.S. Borges, L.N. Epele, and H. Fanchiotti, Phys. Rev. A 38 (1988) 3101; C. Stuckeno and D.H. Kobe, Phys. Rev. A 34 (1986) 3565; M. Mijatovic, B. Veljanoski, and D. Hajdukovic, Hadronic J. 7 (1984) 1207; B.W. Huang, Z.V. Gu, and S.W. Qiam, Phys. Lett A 142 (1989) 203; M. Razavy, Hadronic J., 6 (1983) 406. 